\title{
Clinical longitudinal standards for height, weight, height velocity, weight velocity, and stages of puberty
}

\author{
J. M. TANNER and R. H. WHITEHOUSE \\ From the Department of Growth and Development, Institute of Child Health, University of London
}

Tanner, J. M., and Whitehouse, R. H. (1976). Archives of Disease in Childhood, 51, 170. Clinical longitudinal standards for height, weight, height velocity, weight velocity, and stages of puberty. New charts for height, weight, height velocity, and weight velocity are presented for clinical (as opposed to population survey) use. They are based on longitudinal-type growth curves, using the same data as in the British 1965 growth standards.

In the velocity standards centiles are given for children who are early- and latematuring as well as for those who mature at the average age (thus extending the use of the previous charts). Limits of normality for the age of occurrence of the adolescent growth spurt are given and also for the successive stages of penis, testes, and pubic hair development in boys, and for stages of breast and pubic hair development in girls.

Growth standards based only on cross-sectional survey data are different in form from standards which represent individual longitudinal curves of growth. The two are not interchangeable over the ages at which the adolescent growth spurt may occur, that is from about 7 years onwards in girls and 9 years onwards in boys. The difference is particularly marked in standards for growth velocity. The longitudinal-type standards are those which should be used in following the individual child, whether in the paediatric or adolescent clinic or as a routine monitoring procedure in healthy children. The cross-sectional values are the appropriate ones to use in making comparisons between population groups each studied only in cross-sectional surveys. They give misleading assessments for children followed individually due to the distortion introduced by some children having their adolescent growth spurt early and others late. Mixed longitudinal and cross-sectional data require a more sophisticated combination of these two approaches (Tanner, 1951).

When we first introduced longitudinal-type standards for clinical use (Tanner, Whitehouse, and Takaishi, 1966) we left the more familiar cross-

Received 22 April 1975. sectional centiles also on the charts. Thus in the 'distance' charts, that is those for height or weight attained at each age, the conventional cross-sectionally-derived centiles were indicated by the lines marked $3,10,25,50,75,90,97$ th centiles. The longitudinal-type centiles were indicated by gray-shaded bands. This made the charts look more complicated than they really were, and even introduced the possibility that a clinician might mistakenly interpret a child's growth progress in relation to the wrong set of centiles. Indeed, the latest edition of a standard textbook (Lowrey, 1973) has fallen into this error with regard to the velocity charts and gives (p. 84) quite erroneous values for the average velocities at puberty. Since the charts are used primarily in clinical applications we have now redrawn them so that the longitudinal curves are emphasized, and the cross-sectional information is reduced to a minimum.

This has the added advantage that in the velocity charts we can incorporate new and important information, by giving limits indicating the normal range of ages at which the adolescent growth spurt occurs, and the range of growth velocities appropriate to early-maturing and late-maturing children At the same time we have added to the 'distance' charts limits for the ages at which successive stages 


$$
\text { Height, weight, height velocity, weight velocity, and stages of puberty }
$$

of pubertal sexual development occur in the normal population. The charts illustrated here are confined to one sex only, for reasons of space. ${ }^{\star}$

\section{Charts}

'Distance' charts of height and weight for age. Figs. 1 and 2 show the new charts of height and weight for age in boys. The same data have been used as previously (i.e. British children, 1965). The centiles now refer to boys followed longitudinally: thus the 50th centile line is followed by the boy who has his adolescent spurt at the average age for his population, besides being of average height both before the spurt begins and as an adult, after it has ended. In these charts the shaded areas above the 97th and below the 3rd longitudinal centiles represent the limits of these centiles in cross-sectional data. Thus on the first occasion when a boy is seen his height may fall in these shaded areas without his being outside the conventional 3rd-97th limits for his population; he is large and/or early-maturing or small and/or latematuring. On subsequent occasions, however, such a boy's curve should head towards the longitudinal centile limits and end within them.

The puberty stage standards shown in the chart are referred to below.

Velocity charts of height and weight. Dropping the cross-sectional type elements of the old chart has enabled us to introduce a very important piece of information without overburdening the chart. This relates to the limits of normality for earliness or lateness of maturing, and the characteristics of the adolescent spurt to be expected if a child is at one limit or the other.

Fig. 3 shows the longitudinal centiles for height velocity calculated, as previously, over the period of a whole year and centred about the year of maximum or peak height velocity (Tanner et al., 1966). The labelled centile lines characterize boys with their peak velocity at precisely the average age for this event. The upper panel of Fig. 3 shows additionally, by the hatched area, the form these centiles take in boys who have their peaks at the early limit of 'permissable' age, that is, at the mean age less 2 SDs. (The age of peak is distributed in gaussian fashion so the $S D$ and centile limits are equivalent.) In the early-maturers the peak height velocity tends

\footnotetext{
*The charts may be obtained from Creaseys Ltd., Castle Mead, Hertford, Herts., reference numbers LBH1A, LB HV2A, LGH3A, LGHV4A, LBW5A, LBWV6A. LGW7A, LGW8A, but not from the authors.
}

to be greater than in boys who mature at the average age. Thus for the boys who have their peaks 2 years (roughly $2 \mathrm{SD}$ ) earlier than average, the whole-year peak velocities are 13.1, 10.9, 8.6 $\mathrm{cm} / \mathrm{yr}$ for a 97 th centile spurt, an average spurt, and a 3rd centile spurt.

In the lower panel of Fig. 3 the hatched area characterizes the late maturers, at the same $2 \mathrm{SD}$ limit. Here the peak values are less, being $10 \cdot 3$, $8 \cdot 1$, and $5 \cdot 8 \mathrm{~cm} / \mathrm{yr}$ for 97 th centile peak, average peak, and 3rd centile peak.

In Fig. 4 the new velocity chart is shown in full. The shaded area is produced by sliding the hatched area of the upper part of Fig. 3 over to the position of the hatched area in the lower part of Fig. 3, and clearing the central portion representing the average-age centiles. Thus the shaded area in Fig. 4 represents the limits within which all normally-developing boys' height velocity curves should fall. The white line separates the rising curve area of late maturers (above and to the left) from the falling curve area of early maturers (below and to the right). The 97th, 50th, and 3rd centiles for peak velocity at $2 \mathrm{SD}$ of age early and $2 \mathrm{SD}$ of age late are indicated by the arrow and diamond symbols.

In Fig. 5 a plot is given of an early-maturing boy and a plot of a late-maturing one. Their heights were measured every 6 months and whole-year velocities plotted on each occasion, the centre of the plot being at the central age of the whole year. (Only annual measurements were available, however, from age 14 onwards.) The early maturer had a peak height velocity at about the 50 th centile for his age at peak; the late maturer had a peak at about the 25th centile for his age at peak. Both curves are within normal limits, though only just.

Fig. 6 shows the weight velocity standards for boys, constructed in the same way as for height. The regression of peak velocity on age at peak is lower for weight than for height (Tanner et al., 1966).

The charts for girls are similar to those for boys. In girls, however, the regression of peak height velocity on age at peak is less than that for boys, so there is less difference between the peaks of early and late maturers than in boys. In girls the corresponding weight regression is zero.

Relation between distance and velocity standards. There is a difference between our presentations of distance and velocity standards which unless made explicit might in some cases be confusing. The centile lines shown in the distance 


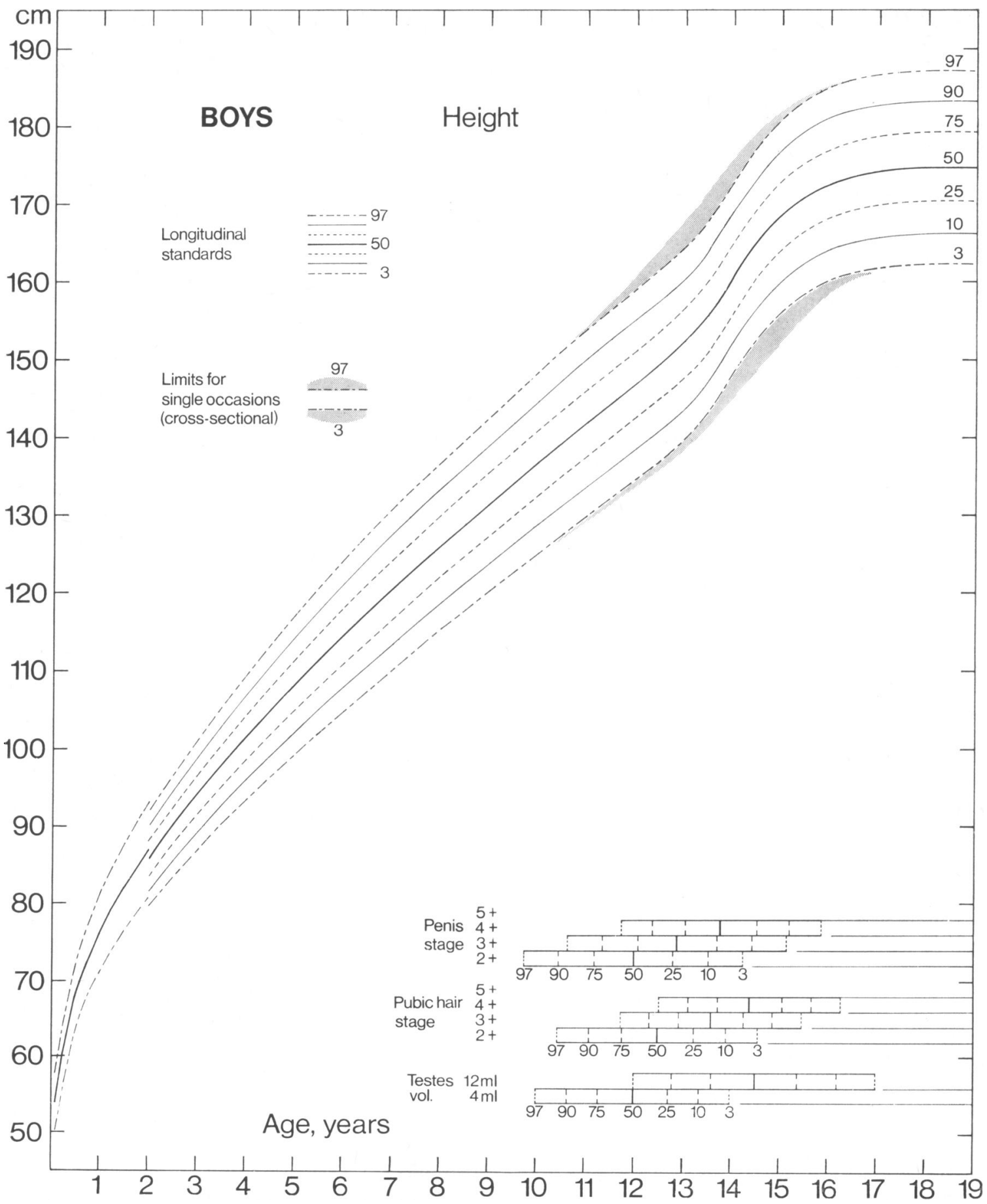

FIG. 1.-Longitudinal standards for height attained at given age, boys. The shaded areas represent the 97th and 3rd centile limits of cross-sectionally derived standards. 
Height, weight, height velocity, weight velocity, and stages of puberty

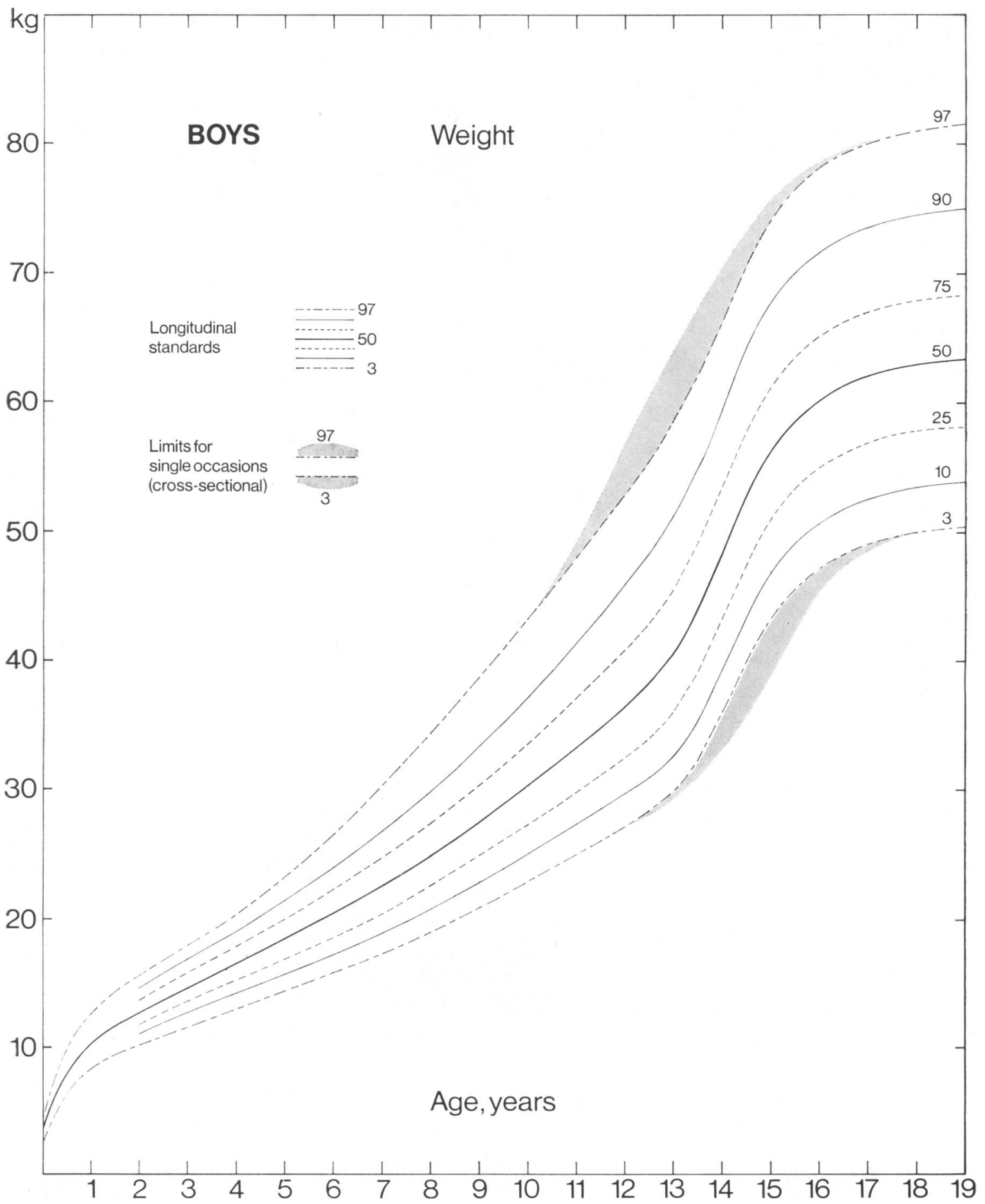

Fig. 2.-Longitudinal standards for weight attained at given age, boys. The shaded areas represent the 97th and 3rd centile limits of cross-sectionally derived standards. 

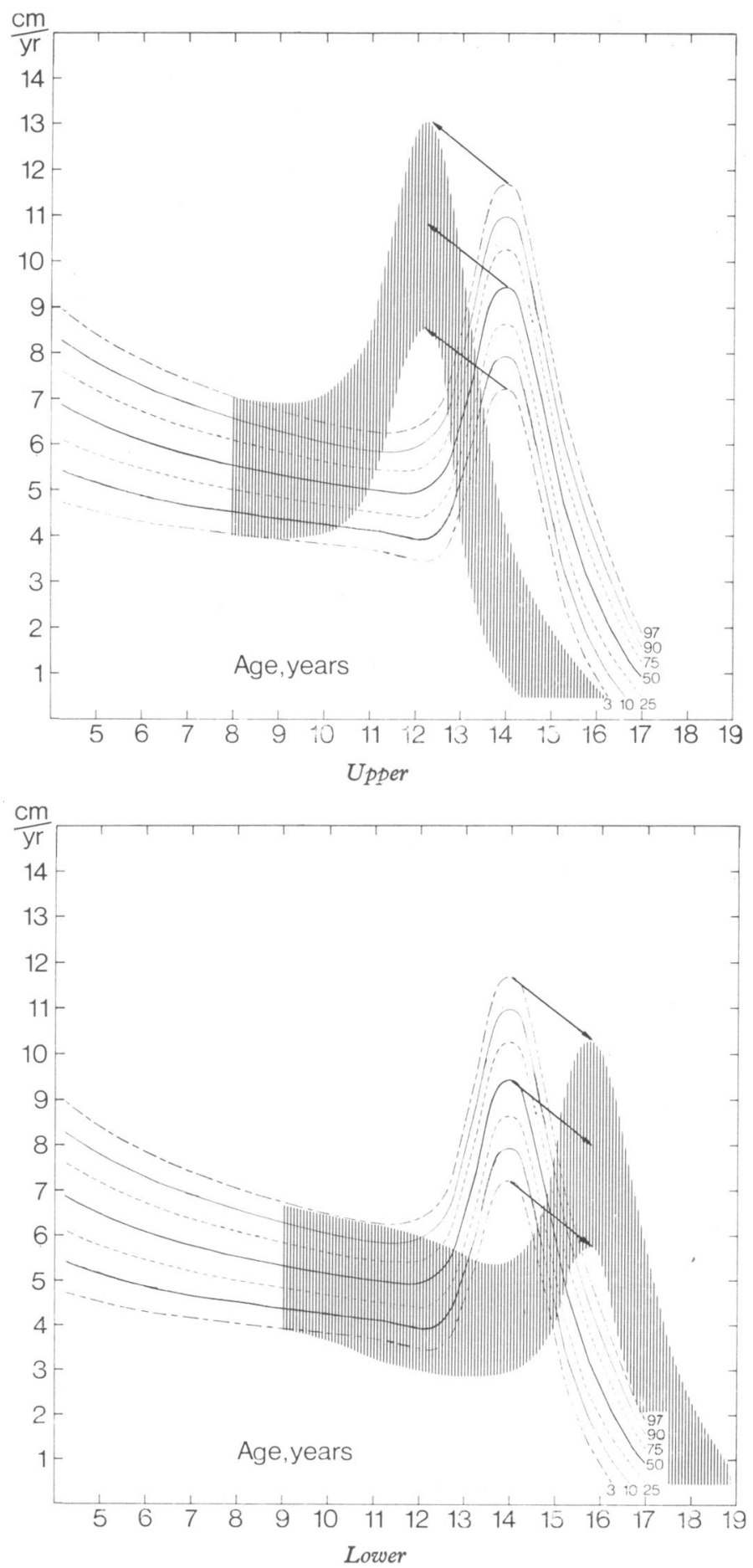

FIG. 3.-Construction of longitudinal standards for height velocity. Centiles represent boys having peak velocity at average age of peak. Upper, hatched area represents centiles for boys with peak occurring 2 SDs of age before mean age (approximately 2 years early). Lower, hatched area represents centiles for boys with peak occurring 2 SDs after mean age (approximately 2 years late). 


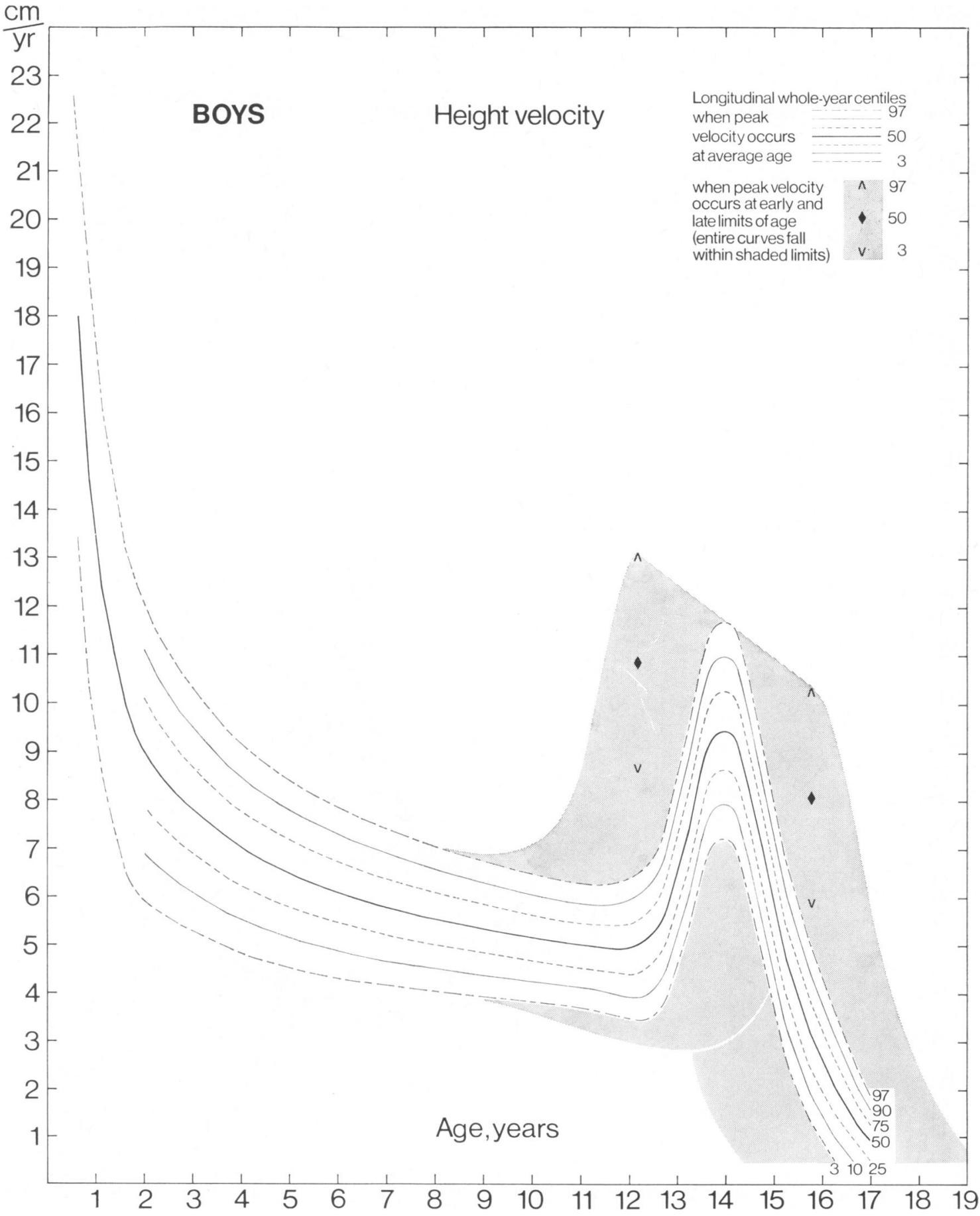

FIG. 4.-Longitudinal standards for height velocity, boys. Shaded area represents total of hatched areas in Fig. 3 upper and lower; it thus encloses all velocity curves within 3rd-97th centile limits for age and for peak velocity. 3rd, 50th, and 97th centiles for early-and late-maturing boys are indicated by arrowhead and diamond symbols. 


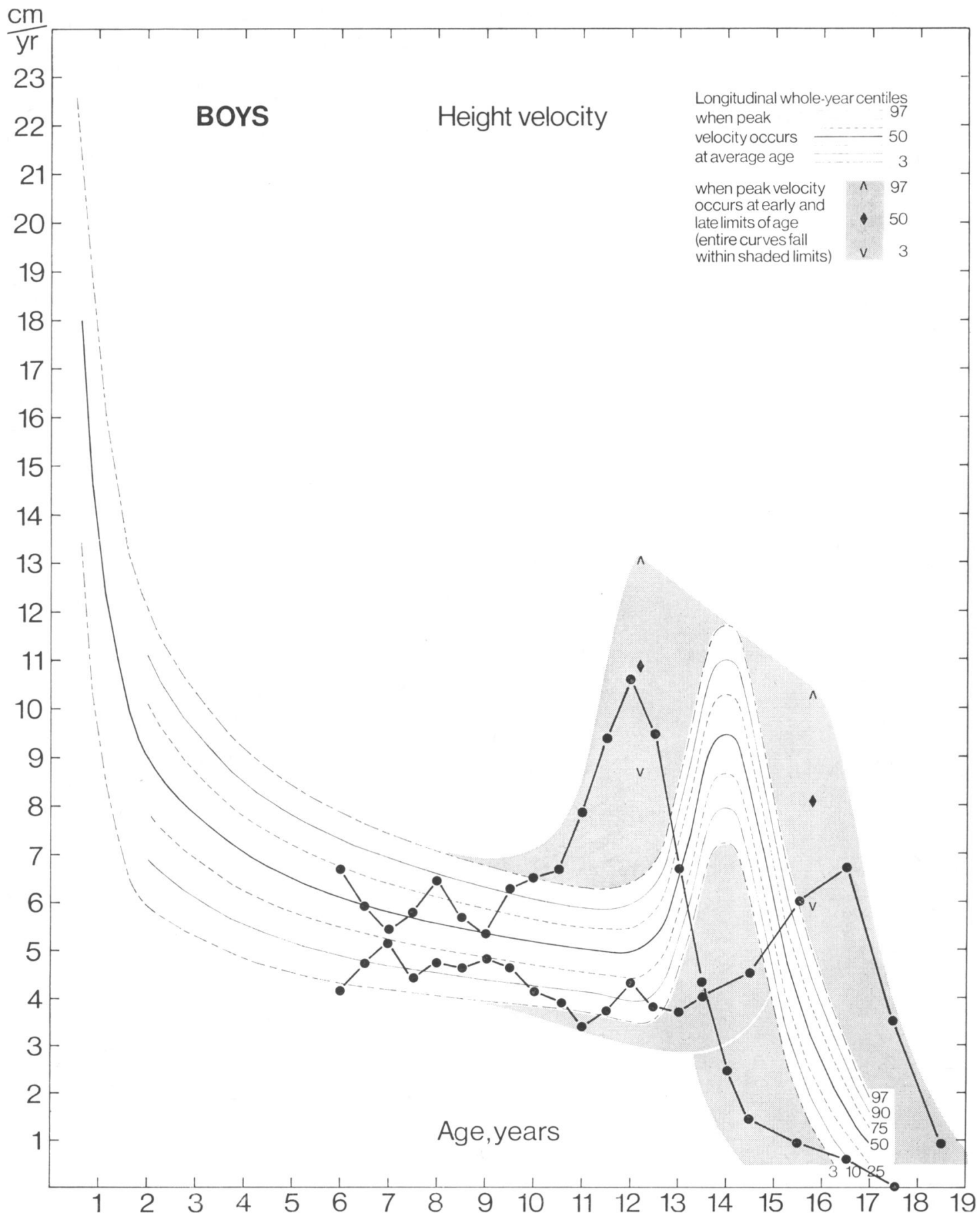

FIG. 5.-Height velocities of an early and a late maturing boy. Whole-year velocities plotted every 6 months, at central point of last completed year, except from age 14 onwards, where only annual measurements were available. 


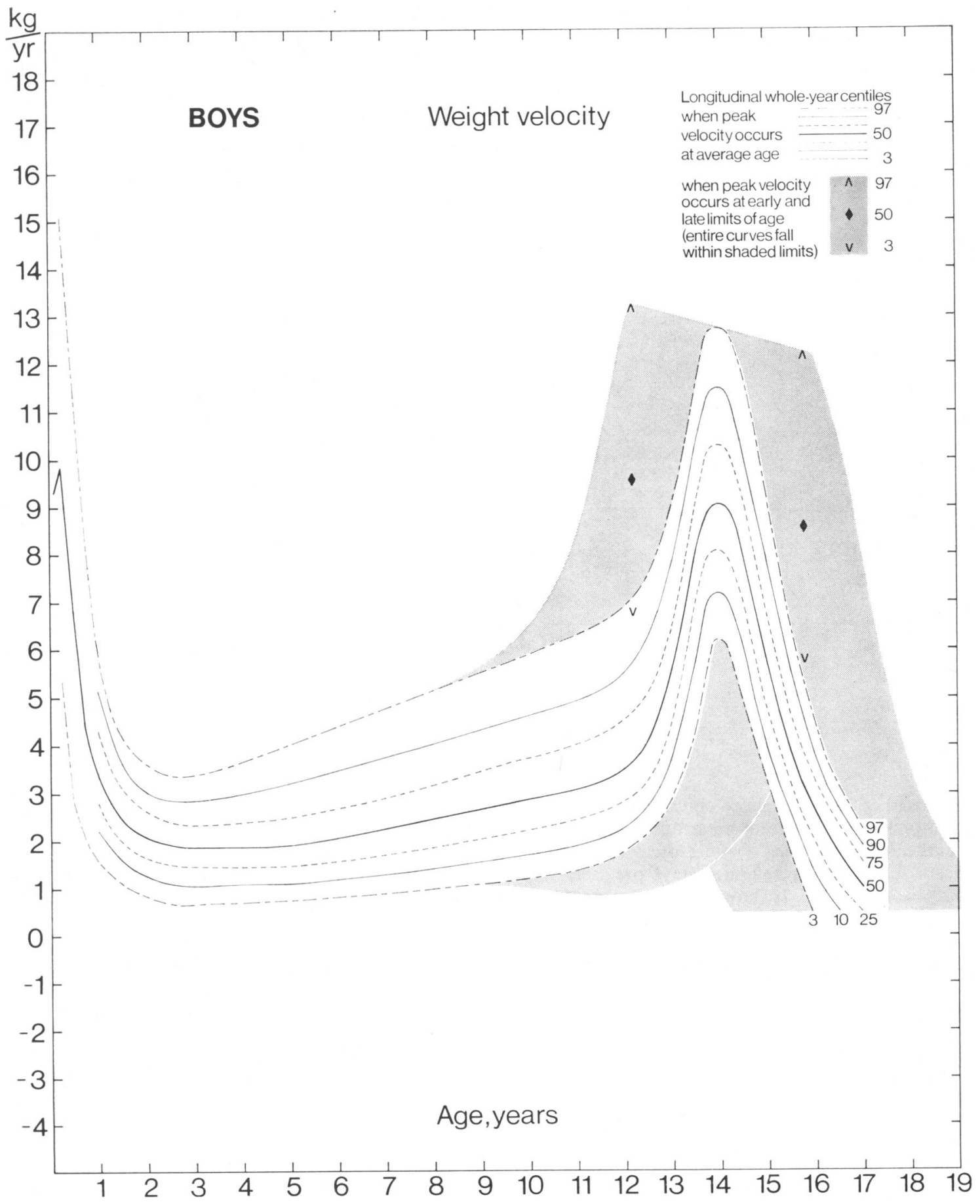

FIG. 6.-Longitudinal standards for weight velocity, boys. Shaded areas as for Fig. 4. 


\section{Centiles for age in given pubertal stages}

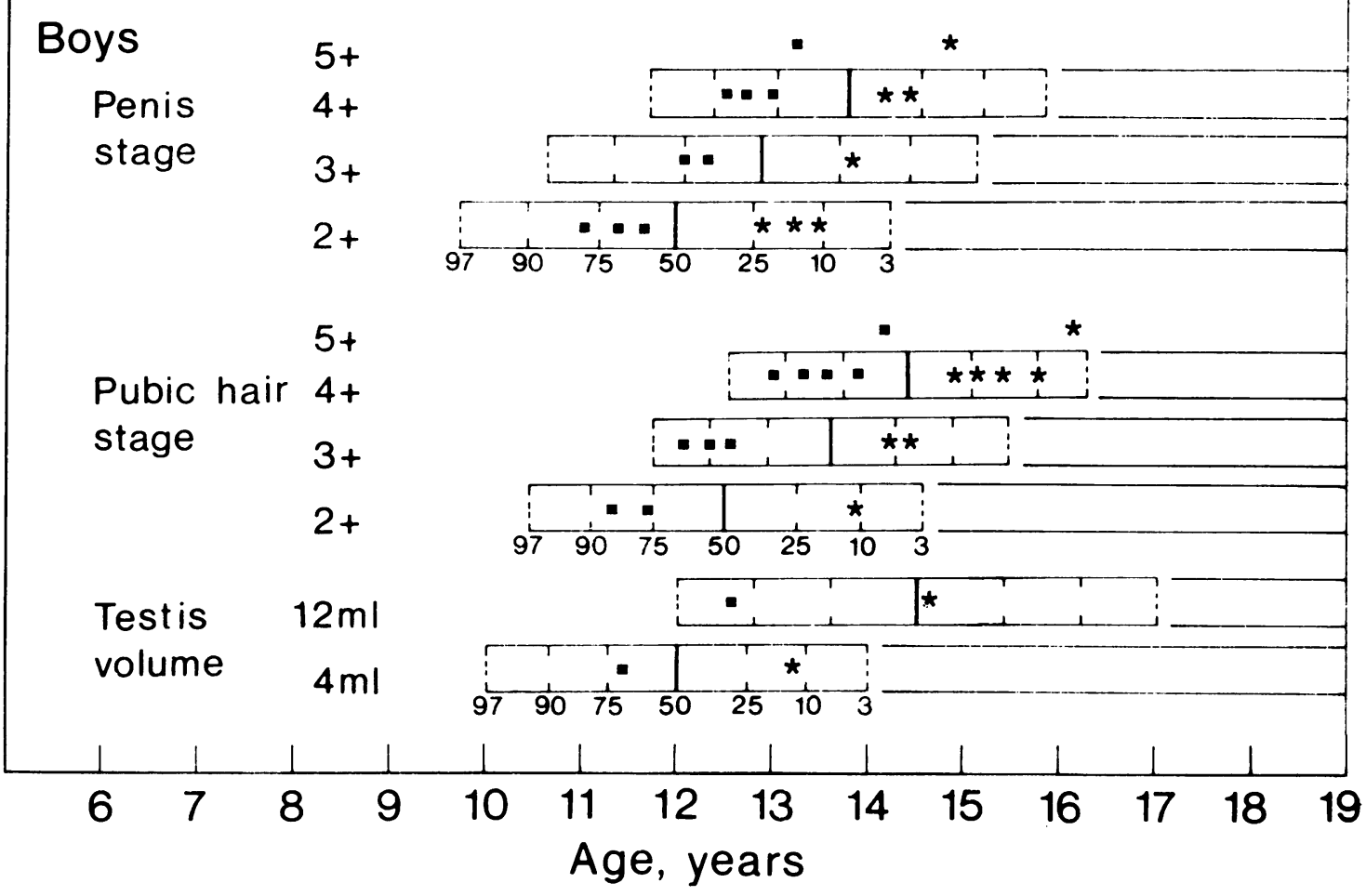

FIG. 7.-A generally early-maturing boy $(\square)$ and a generally late-maturing $\left.{ }^{\star}{ }^{\star}\right)$ boy plotted at successive ages on the puberty standards.

standard of Fig. 1 relate to children who have their peak velocities at the average time. Thus a 90th centile child who has a spurt of average intensity occurring at the average age stays precisely on his 90th centile line. If, however, he has a late spurt he will fall in centile status until his spurt begins, and then catch up. It would in principle be possible to put in a shaded area within which the distance curves of early- and late-maturing large and small boys would lie. However, such an area would be a good deal wider than that shown in our actual Fig. 1. This is because in normal children final adult height is practically independent of earliness or lateness of maturation. Thus a child who is at the 3rd centile for final height and the 3rd centile for maturing (i.e. late) will lie at the $0.03 \times 0.03=0.009$ probability level for the reference population when at his lowest point, and this is considerably below the cross-sectionallyderived 3rd centile shown by the shading. (He would in fact be a case of the small/delay diagnosis (Tanner, 1973).)

Such a child, however, would usually have a velocity curve entirely within the normal limits given in Fig. 4. The peak would be late, of course, but because this 'distance' or height is low it does not follow that his velocity should be low also. There is considerable independence between the amount gained during the adolescent spurt and the final height attained. Indeed a 3rd centile child starting the spurt either late or at the average time must have a nearly average spurt in order to end up at the 3rd centile as an adult. Thus there is no actual contradiction in a child being outside the distance but inside the velocity standards.

Puberty stage standards. In Fig. 1 normal limits for stages of puberty are shown. The penis (previously called 'genitalia') and pubic hair (PH) stages are those described by Tanner $(1962,1973)$. 
The terminology is as follows. The designation $\mathrm{PH} 2$ represents the instant when the appearance PH2 first becomes visible. This is, of course, seldom observable; what the doctor sees is a boy who has attained the appearance PH2 but not yet the appearance PH3. We say this boy is in the stage $\mathrm{PH} 2+$, and we give the limits of ages in the reference population within which the stage $\mathrm{PH} 2+$ is found. It is quite arbitrary which end of the age limit is called the 97th centile and which the 3rd; we have preferred to designate early-maturing as 97th and late-maturing as 3rd. Stage 5 has no age limits since it persists during adult life; it should, of course, be entered before the age of the late limit for stage $4+$ is reached. A child may be plotted on the graph each time he is seen; Fig. 7 shows such a plot for one boy who is generally early and another who is generally late.

The testes volume is measured by the Prader orchidometer (Zachmann et al., 1974) in which a series of testes models of volumes $2,3,4,6,8,10,12$, 15,20 , and $25 \mathrm{ml}$ are compared tactually with the actual testis. We have selected the two 'levels' of volume, $4 \mathrm{ml}$ and $12 \mathrm{ml}$, for plotting, the former because it represents the first clear sign of pubertal increase in size and the latter because it is a volume reached in mid or late puberty by all normal boys.

It is essential that standards for pubic hair and penis (or genitalia) growth are given separately, since often the two develop with different timings (Marshall and Tanner, 1970). The same applies to standards for breast and pubic hair development in girls (Marshall and Tanner, 1969).
A fuller account of the manner in which these standards were constructed will be published elsewhere. The age limits represent, as best we can determine it, the situation in British children in the period 1965-1970.

We are most grateful for the helpful comments of Dr. W. A. Marshall, Mr. M. J. R. Healy, and Mr. N. Cameron; and for financial support from the Medical Research Council, the Nuffield Foundation, and the Department of Health and Social Security.

\section{REPBRENCES}

Lowrey, G. H. (1973). Growth and Development of Children, 6th ed. Yearbook Medical Publishers, Chicago.

Marshall, W. A., and Tanner, J. M. (1969). Variation in pattern of pubertal changes in girls. Archives of Disease in Childhood, 44, 291.

Marshall, W. A., and Tanner, J. M. (1970). Variations in the pattern of pubertal changes in boys. Archives of Disease in Childhood, 45, 13.

Tanner, J. M. (1951). Notes on the reporting of growth data. Human Biology, 23, 93.

Tanner, J. M. (1962). Growth at Adolescence, 2nd ed. Blackwell, Oxford.

Tanner, J. M. (1973). Physical growth and development. Textbook of Paediatrics, p. 224. Ed. by J. O. Forfar and G. C. Arneil. Churchill Livingstone, Edinburgh and London.

Tanner, J. M., Whitehouse, R. H., and Takaishi, M. (1966). Standards from birth to maturity for height, weight, height velocity and weight velocity: British children, 1965. Archives of Disease in Childhood, 41, 454 and 613.

Zachmann, M., Prader, A., Kind, H. P., Häfliger, H., and Budliger, H. (1974). Testicular volume during adolescence. Helvetica Paediatrica Acta, 29, 61.

Correspondence to Professor J. M. Tanner, Department of Growth and Development, Institute of Child Health, 30 Guilford Street, London WC1N 1EH. 